\title{
A POSTERIORI ERROR ESTIMATES FOR DISCONTINUOUS GALERKIN METHODS FOR THE GENERALIZED KORTEWEG-DE VRIES EQUATION
}

\author{
OHANNES KARAKASHIAN AND CHARALAMBOS MAKRIDAKIS
}

\begin{abstract}
We construct, analyze and numerically validate a posteriori error estimates for conservative discontinuous Galerkin (DG) schemes for the Generalized Korteweg-de Vries (GKdV) equation. We develop the concept of dispersive reconstruction, i.e., a piecewise polynomial function which satisfies the GKdV equation in the strong sense but with a computable forcing term enabling the use of a priori error estimation techniques to obtain computable upper bounds for the error. Both semidiscrete and fully discrete approximations are treated.
\end{abstract}

\section{INTRODUCTION}

We consider the initial-boundary value problem (IBVP) for the GKdV equation

$$
\left\{\begin{aligned}
u_{t}+\left(u^{p+1}\right)_{x}+\epsilon u_{x x x} & =0, & & 0<x<1, t>0 \\
u(x, 0) & =u^{0}(x), & & 0<x<1,
\end{aligned}\right.
$$

with periodic boundary conditions on the interval $[0,1]$ where $\epsilon$ is a positive parameter.

The particular equations in (11) are part of a more general class which have arisen in recent years as, e.g., approximate models for the unidirectional propagation of waves in a variety of nonlinear, dispersive media; cf. [8 10,24. The equations in (11) can be seen as an important case of dispersive approximations of conservation laws, i.e., of equations

$$
u_{t}+f(u)_{x}+\epsilon u_{x x x}=0 .
$$

Such equations have attracted increasing attention as testbeds for the competition between nonlinear and dispersive effects, and their accurate numerical approximation is an interesting and challenging problem with several applications.

The literature on numerical methods for the $\mathrm{KdV}$ equation $(p=1)$ is vast, including finite difference, finite element and spectral methods; see e.g., [1, 4, [16], 25], 28], 29], 30], 31, [5], 15], [18, [26], 27].

More recently, discontinuous Galerkin formulations for the $\mathrm{KdV}$ and other nonlinear dispersive equations began appearing. This is not surprising given the general high level of interest in such methods. Indeed, DG methods combine advantages of

Received by the editor February 5, 2013 and, in revised form, August 8, 2013.

2010 Mathematics Subject Classification. Primary 65M12, 65M60; Secondary 35Q53.

The work of the first author was partially supported by NSF grants DMS-0811314 and NSF1216740 .

The work of the second author was supported by EU program FP7-REGPOT-2009-1, grant 245749 through the Archimedes Center for Modeling, Analysis and Computation (ACMAC) of the Department of Applied Mathematics at the University of Crete and by GSRT grant 1456. 
both finite element and finite volume methods, in particular, high order accuracy, flexibility and discrete conservation. Runge-Kuta DG methods for hyperbolic conservation laws are probably the most popular class of finite element methods for such problems; see e.g. 14]. Recently, there has been intense activity on evolution equations with high-order derivatives [13, 32, 33. In particular in the work [13 a class of DG methods for (1) is introduced and analyzed. Recently 11 introduced a different class of DG methods for (1) which are designed to satisfy key discrete versions of its conservation properties. The schemes preserve the first two invariants (the integral and $L^{2}$ norm) of the numerical approximations. Numerical experiments exhibited in [11] have provided strong evidence that this property imparts the approximations with beneficial attributes such as more faithful reproduction of the amplitude and phase of traveling wave solutions as well as linear growth of the errors as a function of time.

In the present paper we analyze the methods of [11,13] from the a posteriori point of view. Our main result (Theorem 5.1) consists in showing that the errors between the solution of (1) and the fully discrete approximation is bounded from above by a computable quantity. To the best of our knowledge this is the first result of its kind for the GKdV equation and for any numerical method extant. The main tool used in the development of the a posteriori error indicators and the accompanying analysis is a spatial reconstruction operator corresponding to the third-order differential operator in (1). Using this operator, one constructs a function, the dispersive reconstruction, which is twice continuously differentiable, and satisfies the GKdV equation (1) in the strong sense but with a computable forcing term.

The idea of using elliptic reconstruction operators in the context of a posteriori error estimates for parabolic equations was introduced by Makridakis and Nochetto in [22] and subsequently developed in a series of papers, e.g., 7, 17, 20,21]; see also [3, 23. There are significant differences, however, between the present development and the above mentioned works. For one, the PDE considered is nonlinear and more importantly, the third derivative operator lacks the coercivity of the operator $-\Delta$. Furthermore, the reconstructed function in the above works belongs to the "energy" space thus requiring an approximation in the finite element space. In our case, the reconstructed function is itself a piecewise polynomial function, albeit of higher degree than those used in the approximations, is (globally) twice differentiable and periodic and can be easily computed. The fact that the reconstructed function is sufficiently smooth to satisfy the PDE (1) with a computable forcing term makes it possible to obtain the a posteriori error estimates using PDE techniques. It is worth noting that this same feature of our approach eliminates the constraint of working solely with test functions which are in the finite element space.

A further important attribute of our approach to reconstruction is that it can be applied to derive a posteriori error estimates for a host of other dispersive (or even nondispersive) equations possessing higher order spatial derivatives. Last but not least, it can be adapted to handle different DG formulations and, in particular, the formulation of 13 .

It must be said that in the presence of nonlinearites and especially those involving a spatial derivative, the accurate determination of the sharpness of the a posteriori error estimators as a function of the spatial and temporal discretization parameters 
becomes a subtle and difficult issue that we do not directly address in the present work; however, see Remarks 4.1, 4.2 and Table 1.

The paper is organized as follows: Section 2 begins with preliminaries and gives a summary of some key results from [11] upon which the present development is based. In Section 3 we introduce the dispersive reconstruction operator $\mathcal{R}$, prove existence and uniqueness as well as some of its important properties. Section 4 is devoted to deriving a posteriori error estimates for the semidiscrete formulation introduced in 11. In Section 5 we develop a posteriori error estimates for fully discrete approximations generated by the Implicit Euler method. In Section 6 we provide an outline showing that a similar treatment can be applied to the method of Y. Cheng and C.-W. Shu. Finally, Section 7 is devoted to numerical experiments showing the behaviour of the various error indicators. In particular, we provide evidence that the upper bounds supplied by the a posteriori error estimates appear to converge to zero, as a function of the temporal discretization parameter, at the same linear rate of the Implicit Euler method.

\section{Preliminaries}

2.1. The meshes. Let $\mathcal{T}_{h}$ denote a partition of the real interval $[0,1]$ of the form $0=x_{0}<x_{1}<\cdots<x_{M}=1$. We will also say that $\mathcal{T}_{h}$ is a mesh on [0,1]. The points $x_{m}$ are called nodes while the intervals $I_{m}=\left[x_{m}, x_{m+1}\right]$ will be referred to as cells. The notation $x_{m}^{-}=x_{m}^{+}=x_{m}$ will be useful in taking account, respectively, of leftand right-hand limits of discontinuous functions. The caveat followed throughout is that $x_{0}^{-}=x_{M}^{-}$and $x_{M}^{+}=x_{0}^{+}$corresponding to the underlying spatial periodicity of the solutions being approximated.

It should be noted at this point that there is no need to assume global quasi uniformity of the spatial mesh in the derivation of the a posteriori estimates. This is in contrast to the situation where a priori estimates were developed as in [11,13. Instead, it is sufficient to assume a local mesh condition whereby the lengths $h_{I}, h_{J}$ of any two adjacent cells are related by $1 / \alpha \leq h_{I} / h_{J} \leq \alpha$ for some $\alpha>0$ which may be chosen arbitrarily.

2.2. Function spaces. In addition to the usual Sobolev spaces $W^{s, p}=W^{s, p}([0,1])$, repeated use will be made of the so-called broken Sobolev spaces $W^{s, p}\left(\mathcal{T}_{h}\right)$. These are the finite Cartesian products $\Pi_{I \in \mathcal{T}_{h}} W^{s, p}(I)$. Note that if $s p>1$, the elements of $W^{s, p}\left(\mathcal{T}_{h}\right)$ are uniformly continuous when restricted to a given cell, but they may be discontinuous across nodes. For the purpose of quantifying these potential discontinuities, introduce the following notation: for $v \in W^{s, p}\left(\mathcal{T}_{h}\right), s \geq 1$, let $v_{m}^{+}$ and $v_{m}^{-}$denote the right-hand and left-hand limits, respectively, of $v$ at the node $x_{m}$. The jump $\left[v_{m}\right]$ (sometimes written $[v]_{m}$ ) of $v$ at $x_{m}$ is defined as $v_{m}^{+}-v_{m}^{-}$. Similarly, the average $\left\{v_{m}\right\}$ (also denoted $\{v\}_{m}$ ) of $v$ at $x_{m}$ is $\frac{1}{2}\left(v_{m}^{+}+v_{m}^{-}\right)$. These are all standard notations in the context of DG methods. In all cases, the definitions are meant to adhere to the convention that $v_{0}^{-}=v_{M}^{-}$and $v_{M}^{+}=v_{0}^{+}$. In particular, according to this convention $[v]_{0}=[v]_{M}=v_{0}^{+}-v_{M}^{-}$and $\{v\}_{0}=\{v\}_{M}=\frac{1}{2}\left(v_{0}^{+}+v_{M}^{-}\right)$.

For an interval $I$ (e.g. $I=[0,1]$ or $I \in \mathcal{T}_{h}$ ) norms in the Sobolev classes $W^{s, p}(I)$ will be denoted $\|\cdot\|_{W^{s, p}(I)}$ or $\|\cdot\|_{s, p, I}$. We shall omit $s$ when it is equal to zero, $p$ when it is equal to two and $I$ when it is equal to $[0,1]$. Also, single vertical bars $|\cdot|_{s, p, I}$ will be used to denote the corresponding seminorms. Another item of notation that will be used throughout is $(u, v)_{I}=\int_{I} u v d x, \quad(u, v)=\int_{0}^{1} u v d x$. 
Use will also be made of the classes $L^{p}\left([0, T] ; W^{s, r}\right)$ of functions $u=u(x, t)$ which are measurable mappings from $[0, T]$ into $W^{s, r}$ and such that

$$
\|u\|_{L^{p}\left([0, T] ; W^{s, r}\right)}=\left(\int_{0}^{T}\|u(\cdot, \tau)\|_{W^{s, r}}^{p} d \tau\right)^{1 / p}<\infty
$$

with the usual modification if $p=\infty$.

We shall also make use of spaces of smooth and periodic functions. Henceforth, $C^{m}[0,1], m \geq 0$ will denote the space of functions $u$ whose derivatives of order up to $m$ are continuous on $[0,1]$ and satisy the periodicity conditions $u^{(j)}(0)=$ $u^{(j)}(1), j=0, \ldots, m$.

The following, basic embedding inequality (see 2]) will find frequent use in our development. For $v \in H^{1}\left(\mathcal{T}_{h}\right)=W^{1,2}\left(\mathcal{T}_{h}\right)$ and any cell $I \in \mathcal{T}_{h}$, there is a constant $c$ which is independent of the cell $I$ such that

$$
\|v\|_{\infty, I} \leq c\left(h_{I}^{-1 / 2}\|v\|_{I}+h_{I}^{1 / 2}\left\|v_{x}\right\|_{I}\right),
$$

where $h_{I}$ is the length of $I$. Indeed, the dependence of (3) on $h_{I}$ is easily ascertained by a simple scaling argument. Note that (3) may also be viewed as a trace inequality.

2.3. The discontinuous polynomial spaces. The spatial approximations will be sought in the space of discontinuous, piecewise polynomial functions $V_{h}^{q}$ subordinate to the mesh $\mathcal{T}_{h}$, viz.

$$
V_{h}^{q}=\left\{v:\left.v\right|_{I} \in \mathcal{P}_{q}(I), I \in \mathcal{T}_{h}\right\},
$$

where $\mathcal{P}_{q}$ is the space of polynomials of degree $q$ and $q \geq 2$. For $I \in \mathcal{T}_{h}, V_{h}^{q}(I)$ will denote the subspace of $V_{h}^{q}$ of functions that vanish outside of $I$. In this regard, we shall often find it convenient to use the characteristic function $\chi_{I}$ of the cell $I$.

The spaces $V_{h}^{q}$ have well-known, local approximation and inverse properties which are spelled out here for convenience; cf. 6], 12. Let $q \geq 0$ be fixed and let $v \in H^{q+1}(I)$. Then there exists a $\chi \in \mathcal{P}_{q}(I)$ such that

$$
|v-\chi|_{j, I} \leq c h_{I}^{q+1-j}|v|_{q+1, I}, \quad 0 \leq j \leq q+1,
$$

where $|v|_{i, I}$ denotes the seminorm $\left\|v^{(i)}\right\|_{L^{2}(I)}$ on the Sobolev space $H^{i}(I)$ and the constant $c$ is independent of $h_{I}$. The above property continues to hold if the $L^{p}$ based Sobolev spaces replace the $L^{2}$-based classes $H^{j}$. In particular, it holds for the $L^{\infty}$ norm, which is to say, there is a $\chi \in \mathcal{P}_{q}(I)$ such that

$$
\left|\partial_{x}^{j}(v-\chi)\right|_{L^{\infty}(I)} \leq c h_{I}^{q+1-j}\left|\partial_{x}^{q+1} v\right|_{L^{\infty}(I)}, \quad 0 \leq j \leq q+1 .
$$

The equally well-known inverse inequality

$$
|\chi|_{j, I} \leq \operatorname{ch}_{I}^{-j}|\chi|_{0, I}, \quad 0 \leq j \leq q+1
$$

for all $\chi \in \mathcal{P}_{q}(I)$ will also find frequent use.

2.4. The weak formulation. In this section we exhibit the basic weak formulations that form the basis for the semidiscrete and fully discrete methods contained in 11 . 
On $H^{1}\left(\mathcal{T}_{h}\right) \times H^{1}\left(\mathcal{T}_{h}\right)$ we define the form $\mathcal{N}(\cdot, \cdot)$ by

$$
\mathcal{N}(u, v)=-\sum_{I \in \mathcal{T}_{h}}\left(u^{p+1}, v_{x}\right)_{I}-\sum_{m=0}^{M-1} \hat{\phi}\left(u_{m}^{+}, u_{m}^{-}\right)\left[v_{m}\right]
$$

where, $\hat{\phi}\left(u_{m}^{+}, u_{m}^{-}\right)=\frac{1}{p+2} \sum_{j=0}^{p+1}\left(u_{m}^{+}\right)^{p+1-j}\left(u_{m}^{-}\right)^{j}$.

We shall use the same symbol $\mathcal{N}$ to denote the nonlinear operator $\mathcal{N}: H^{1}\left(\mathcal{T}_{h}\right) \rightarrow$ $V_{h}^{q}$ induced by the above form according to

$$
(\mathcal{N}(u), v)=\mathcal{N}(u, v), \quad \forall v \in V_{h}^{q} .
$$

That the operator $\mathcal{N}$ is well-defined follows easily from the Riesz Representation Theorem.

The following result obtains for this operator.

Lemma 2.1. (i) The form $\mathcal{N}$ defined by (8) is consistent in the sense that for all $u$ in $C^{1}[0,1]$, there holds

$$
\mathcal{N}(u, v)=\left(\left(u^{p+1}\right)_{x}, v\right), \quad \forall v \in H^{1}\left(\mathcal{T}_{h}\right) .
$$

(ii) Furthermore, $\mathcal{N}$ is conservative in the sense that

$$
\mathcal{N}(v, v)=0, \quad \forall v \in H^{1}\left(\mathcal{T}_{h}\right) .
$$

We also define the bilinear form $\mathcal{D}: H^{3}\left(\mathcal{T}_{h}\right) \times H^{3}\left(\mathcal{T}_{h}\right) \rightarrow R$ by

$$
\mathcal{D}(u, v)=\sum_{I \in \mathcal{T}_{h}}\left(u_{x}, v_{x x}\right)_{I}-\sum_{j=0}^{M-1}\left(u_{x x}\left(x_{j}^{+}\right)[v]_{j}-[u]_{j} v_{x x}\left(x_{j}^{+}\right)\right)+\sum_{j=0}^{M-1}\left\{u_{x}\right\}_{j}\left[v_{x}\right]_{j} .
$$

A useful alternative definition of the form $\mathcal{D}$ is

$$
\mathcal{D}(u, v)=-\sum_{I \in \mathcal{T}_{h}}\left(u, v_{x x x}\right)_{I}-\sum_{j=0}^{M-1} u_{x x}\left(x_{j}^{+}\right)[v]_{j}+\sum_{j=0}^{M-1}\left\{u_{x}\right\}_{j}\left[v_{x}\right]_{j}-\sum_{j=0}^{M-1} u_{j}^{-}\left[v_{x x}\right] .
$$

The two definitions are equivalent in the sense that they agree for all $u, v \in H^{3}\left(\mathcal{T}_{h}\right)$.

The form $\mathcal{D}$ induces the linear operator (using the same symbol) $\mathcal{D}: H^{3}\left(\mathcal{T}_{h}\right) \rightarrow$ $V_{h}^{q}$ defined by

$$
(\mathcal{D} u, v)=\mathcal{D}(u, v), \quad \forall v \in V_{h}^{q} .
$$

The following result obtains for the bilinear form $\mathcal{D}$ :

Lemma 2.2. The bilinear form $\mathcal{D}$ defined by (11) (or (12)) is

(i) consistent in the sense that for $u$ in $C^{2}[0,1] \cap H^{3}\left(\mathcal{T}_{h}\right)$ there holds

$$
\mathcal{D}(u, v)=\left(u_{x x x}, v\right), \quad \forall v \in H^{3}\left(\mathcal{T}_{h}\right),
$$

(ii) skew-adjoint (conservative), which is to say,

$$
\mathcal{D}(v, v)=0 \quad \forall v \in H^{3}\left(\mathcal{T}_{h}\right) .
$$

The proofs of the preceding two lemmas can be found in [1]. Furthermore, The following bound will prove useful later in connection with the error estimator term $\mathcal{E}_{3}$ introduced in $\S 5$.

Lemma 2.3. Let $u \in H^{3}\left(\mathcal{T}_{h}\right)$. Then, for each cell $I=\left[x_{m}, x_{m+1}\right], j=0, \ldots, M-$ 1, the following local bound holds:

$$
\|\mathcal{D} u\|_{I}^{2} \leq c\left(\left\|u_{x x x}\right\|_{I}^{2}+h_{m}^{-5}[u]_{m}^{2}+h_{m}^{-3}\left[u_{x}\right]_{m}^{2}+h_{m}^{-3}\left[u_{x}\right]_{m+1}^{2}+h_{m}^{-1}\left[u_{x x}\right]_{m+1}^{2}\right) .
$$


Proof. Integrating the first term on the right side of (12) thrice, we obtain

$$
\begin{aligned}
(\mathcal{D} u, v)= & \sum_{I \in \mathcal{T}_{h}}\left(u_{x x x}, v\right)_{I}+\sum_{j=0}^{M-1}\left[u_{x x}\right]_{j} v_{j}^{-}-\sum_{j=0}^{M-1}\left[u_{x}\right]_{j}\left\{v_{x}\right\}_{j} \\
& +\sum_{j=0}^{M-1}[u]_{j} v_{x x}\left(x_{j}^{+}\right), \quad \forall v \in V_{h}^{q} .
\end{aligned}
$$

Letting $v=(\mathcal{D} u) \chi_{I}$ where $\chi_{I}$ is the characteristic function of $I$, we obtain

$$
\begin{aligned}
\|\mathcal{D} u\|_{I}^{2}= & \left(u_{x x x}, \mathcal{D} u\right)_{I}+\left[u_{x x}\right]_{m+1} v_{m+1}^{-}-\frac{1}{2}\left[u_{x}\right]_{m} v_{x}\left(x_{m}^{+}\right) \\
& -\frac{1}{2}\left[u_{x}\right]_{m+1} v_{x}\left(x_{m+1}^{-}\right)+[u]_{m} v_{x x}\left(x_{m}^{+}\right) .
\end{aligned}
$$

Now using the embedding (3) and inverse (6) inequalities, we get

$$
\max \left\{h_{m}^{1 / 2}\left|v_{m+1}^{-}\right|, h_{m}^{3 / 2}\left|v_{x}\left(x_{m}^{+}\right)\right|, h_{m}^{3 / 2}\left|v_{x}\left(x_{m+1}^{-}\right)\right|, h_{m}^{5 / 2}\left|v_{x x}\left(x_{m}^{+}\right)\right|\right\} \leq c\|v\|_{I} .
$$

Using the Cauchy-Schwarz inequality on the terms in (17) and the bounds contained in (18) keeping in mind that $v=(\mathcal{D} u) \chi_{I}$, the desired bound (15) follows from the arithmetic-geometric mean inequality

Remark 2.1. (i) Note that summing over $I \in \mathcal{T}_{h}$, we obtain the following global version of (15)

$$
\|\mathcal{D} u\|^{2} \leq c \sum_{I \in \mathcal{T}_{h}}\left\|u_{x x x}\right\|_{I}^{2}+c \sum_{m=0}^{M-1}\left(h_{m}^{-5}[u]_{m}^{2}+h_{m}^{-3}\left[u_{x}\right]_{m}^{2}+h_{m}^{-1}\left[u_{x x}\right]_{m}^{2}\right) .
$$

(ii) Another observation is that if $u \in C^{2}[0,1] \cap H^{3}\left(\mathcal{T}_{h}\right)$, then it follows from (16) that $\left.\mathcal{D} u\right|_{I}$ is the $L^{2}$ projection of $\left.u_{x x x}\right|_{I}$ into $V_{h}^{q}(I)$.

2.5. The dispersive projection operator. An important component in the construction and analysis of a priori estimates is the (linear) dispersive projection operator $\mathcal{P}: H^{3}\left(\mathcal{T}_{h}\right) \rightarrow V_{h}^{q}$. For $u \in H^{3}\left(\mathcal{T}_{h}\right)$, let $w:=\mathcal{P} u \in V_{h}^{q}$ be given by

$$
\begin{array}{ll}
(w, v)_{I}=(u, v)_{I}, & \forall v \in \mathcal{P}_{q-3}(I), I \in \mathcal{T}_{h}, \\
w\left(x_{m}^{-}\right)=u\left(x_{m}^{-}\right), & m=1, \ldots, M, \\
\left\{w_{x}\right\}_{m}=\left\{u_{x}\right\}_{m}, & m=0, \ldots, M-1, \text { or } m=1, \ldots, M, \\
w_{x x}\left(x_{m}^{+}\right)=u_{x x}\left(x_{m}^{+}\right), & m=0, \ldots, M-1 .
\end{array}
$$

The following result is easily shown upon using the definition of $\mathcal{P} u$ in the bilinear form $\mathcal{D}$ given by (12). Indeed, the operator $\mathcal{P} u$ was constructed precisely in order for (21) to hold.

Lemma 2.4. The projection $\mathcal{P} u$ defined by (20) satisfies

$$
\mathcal{D} \mathcal{P} u=\mathcal{D} u, \quad \forall u \in H^{3}\left(\mathcal{T}_{h}\right) .
$$

It was proved in [1] that under certain conditions the operator $\mathcal{P}$ is well-defined and possesses good approximation properties. To quote, we have

Proposition 2.1. Assume that $q \geq 2$ is even and that the number of cells in $\mathcal{T}_{h}$ is odd. Then, there exists a unique $w$ satisfying the conditions (20). Furthermore, if 
$u$ is sufficiently smooth, then for $j=0,1$ and $p=2, \infty$, there exists a constant $c$ independent of $h_{I}$ such that

$$
\|u-\mathcal{P} u\|_{W^{j, p}(I)} \leq c h_{I}^{1-j}\left(\sum_{I \in \mathcal{T}_{h}^{N}} h_{I}^{q}\|u\|_{W^{q+1, \infty}(I)}+\sum_{I \in \mathcal{T}_{h} \backslash \mathcal{T}_{h}^{N}} h_{I}^{q+1}\|u\|_{W^{q+2, \infty}(I)}\right),
$$

where $\mathcal{T}_{h}^{N}$ is the set of cells whose length differs from at least one of its two immediate neighbors.

Remark 2.2. It should be noted that the operator $\mathcal{P}$ does not play a role in the construction and analysis of the a posteriori error estimates in this paper. Therefore, the restrictive assumptions of the preceding result are not needed for the development to follow.

\section{The Dispersive ReCONSTRUCTION OPERATOR}

As mentioned earlier, the main tool used in the development of the a posteriori error estimates is a (spatial) dispersive reconstruction operator $\mathcal{R}$ corresponding to the third order differential operator in (11) as well as to the discrete operator $\mathcal{D}$ defined above. In defining the operator $\mathcal{R}$ we are motivated by the following considerations:

(1) For $u \in H^{3}\left(\mathcal{T}_{h}\right), \mathcal{R} u$ should be computable, preferrably locally on each cell

(2) $\mathcal{R} u$ should be globally smooth so that the equality $(\mathcal{R} u)_{x x x}=\mathcal{D} u$ may hold in the strong sense. The objective behind this is the ability to replace the $\mathcal{D} u_{h}$ term in the semidiscrete equation (40) with $(\mathcal{R} u)_{x x x}$. Note that $(\mathcal{R} u)_{x x x}=\mathcal{D} u$ is precisely the first equation of (23).

(3) In deriving a priori estimates, one compares $u_{h}$ to the projection $\mathcal{P} u$ of the solution $u$ of (1). For a posteriori error estimation it turns out that it would be useful to have a reconstruction operator which is a right inverse of $\mathcal{P}$, i.e., $\mathcal{P} \mathcal{R} u=\mathcal{P} u$. Considering the last three equations of the definition of $\mathcal{P}$ in (20) and taking into account the smoothness of $\mathcal{R} u$, we obtain precisely the last three equations of (23).

The dispersive reconstruction corresponding to the form $\mathcal{D}$ is defined in the next theorem.

Theorem 3.1. For each $u \in H^{3}\left(\mathcal{T}_{h}\right)$ there corresponds a unique $\sigma:=\mathcal{R} u \in$ $C^{2}[0,1] \cap V_{h}^{q+3}$ such that for each cell $I=\left[x_{m}, x_{m+1}\right], \quad m=0, \ldots, M-1$ in $\mathcal{T}_{h}$ there holds

$$
\begin{aligned}
\left(\sigma_{x x x}, v\right)_{I} & =(\mathcal{D} u, v), \quad \forall v \in V_{h}^{q}(I), \\
\sigma\left(x_{m}^{+}\right) & =u\left(x_{m}^{-}\right), \\
\sigma_{x}\left(x_{m}^{+}\right) & =\left\{u_{x}\right\}_{m}, \\
\sigma_{x x}\left(x_{m}^{+}\right) & =u_{x x}\left(x_{m}^{+}\right) .
\end{aligned}
$$

Proof. To begin, note that $\sigma$ is defined locally on each cell, hence its existence can be established in a straightforward way. Indeed, let $w \in V_{h}^{q}$ be given by $(w, v)_{I}=(\mathcal{D} u, v) \forall v \in V_{h}^{q}(I)$. The existence of $w$ is a consequence of the Riesz Representation Theorem. Now let $\sigma \in V_{h}^{q+3}$ be the third antiderivative of $w$. The three constants of integration generated can now be chosen so that the remaining three constraints in (23) are satisfied. 
It remains to show that $\sigma$ belongs to $C^{2}[0,1]$. This is arguably the most interesting and important property of the reconstruction operator $\mathcal{R}$. Now for a fixed $I=\left[x_{m}, x_{m+1}\right], m=0, \ldots, M-1$, let $v$ be the characteristic function of $I$. We have

$$
\left(\sigma_{x x x}, v\right)_{I}=\sigma_{x x}\left(x_{m+1}^{-}\right)-\sigma_{x x}\left(x_{m}^{+}\right) .
$$

On the other hand, from the definition of $\mathcal{D}$ we see that in (11) only the terms involving $u_{x x}\left(x_{j}^{+}\right)[v]_{j}$ are nonzero and this for a pair of consecutive indices from the set $\{0,1\}, \ldots,\{M-1,0\}$. Hence, it follows that

$$
(\mathcal{D} u, v)= \begin{cases}-u_{x x}\left(x_{m}^{+}\right)+u_{x x}\left(x_{m+1}^{+}\right), & \text {if } 0 \leq m \leq M-2, \\ -u_{x x}\left(x_{M-1}^{+}\right)+u_{x x}\left(x_{0}^{+}\right), & \text {if } m=M-1 .\end{cases}
$$

Now the fourth equation of (23) stipulates that $\sigma_{x x}\left(x_{m}^{+}\right)=u_{x x}\left(x_{m}^{+}\right)$for each $m$ in the range $0, \ldots, M-1$. Hence comparing (24) and (25) we obtain

$$
\begin{array}{ll}
\sigma_{x x}\left(x_{m+1}^{-}\right)=u_{x x}\left(x_{m+1}^{+}\right)=\sigma_{x x}\left(x_{m+1}^{+}\right), & \text {if } 0 \leq m \leq M-2, \\
\sigma_{x x}\left(x_{M}^{-}\right)=u_{x x}\left(x_{0}^{+}\right)=\sigma_{x x}\left(x_{0}^{+}\right), & \text {if } m=M-1 .
\end{array}
$$

which shows that $\sigma_{x x}$ is continuous on $[0,1]$ and also periodic.

To show that $\sigma_{x}$ is continuous and periodic, we use the test function $v=x-x_{m}$ for $x \in\left[x_{m}, x_{m+1}\right]$ and $v=0$ otherwise. Arguing as above, we obtain

$$
h_{m} \sigma_{x x}\left(x_{m+1}^{-}\right)-\left(\sigma_{x}\left(x_{m+1}^{-}\right)-\sigma_{x}\left(x_{m}^{+}\right)\right)=h_{m} u_{x x}\left(x_{m+1}^{+}\right)+\left\{u_{x}\right\}_{m}-\left\{u_{x}\right\}_{m+1} .
$$

We already showed in (26) that $\sigma_{x x}\left(x_{m+1}^{-}\right)=u_{x x}\left(x_{m+1}^{+}\right)$. Moreover, from (27) and the third equation of (23) it follows that

$$
\sigma_{x}\left(x_{m+1}^{-}\right)=\left\{u_{x}\right\}_{m+1}=\sigma_{x}\left(x_{m+1}^{+}\right),
$$

which shows that $\sigma_{x}$ is continuous and periodic. Finally, to show that $\sigma$ has the same property as its first two derivatives, we use the test function $v=\left(x-x_{m}\right)^{2}$ for $x \in\left[x_{m}, x_{m+1}\right]$ and $v=0$ otherwise. In this case we use (12) to obtain

$$
\begin{aligned}
h_{m}^{2} \sigma_{x x}\left(x_{m+1}^{-}\right)-2 h_{m} \sigma_{x}\left(x_{m+1}^{-}\right) & +2\left(\sigma\left(x_{m+1}^{-}\right)-\sigma\left(x_{m}^{+}\right)\right) \\
=h_{m}^{2} u_{x x}\left(x_{m+1}^{-}\right)-2 h_{m}\left\{u_{x}\right\}_{m+1} & -2 u\left(x_{m}^{-}\right)+2 u\left(x_{m+1}^{-}\right),
\end{aligned}
$$

which implies, after using (26), (28) and the second equation of (23) that

$$
\sigma\left(x_{m+1}^{-}\right)=u\left(x_{m+1}^{-}\right)=\sigma\left(x_{m+1}^{+}\right),
$$

the last equality again following from the the second equation of (23). This concludes the proof.

Remark 3.1. Having shown that $\mathcal{R} u \in C^{2}[0,1] \cap V_{h}^{q+3}$, the first equation of (23) can be expressed as $\sigma_{x x x}=(\mathcal{R} u)_{x x x}=\mathcal{D} u$. We also note that while $\mathcal{R} u$ also satisfies $\mathcal{D} \mathcal{R} u=\mathcal{D} u$ for $u \in H^{3}\left(\mathcal{T}_{h}\right)$, we cannot use it as a substitute to $\mathcal{P} u$ in the a priori analysis since it does not belong to $V_{h}^{q}$. 
3.1. Calculation of $\mathcal{R} u$. In this section we discuss in detail how one can calculate $\sigma=\mathcal{R} u$. We mentioned above that $\sigma$ is defined locally on each cell therefore it can be calculated in parallel. Furthermore, on each cell, $\sigma$ can be calculated efficiently by solving a $(q+4) \times(q+4)$ linear system whose coefficient matrix is invertible and upper triangular.

For this, let $P_{j}(t), j \geq 0$ be the family of Legendre polynomials that are orthogonal on $[-1,1]$, normalized so that $P_{j}(1)=1$. Given a cell $I_{m}=\left[x_{m}, x_{m+1}\right]$, we consider the affine map

$$
x=\frac{h_{m}}{2} t+\frac{x_{m}+x_{m+1}}{2}, \quad-1 \leq t \leq 1,
$$

that maps $[-1,1]$ onto $I_{m}$. Also, consider the family of Legendre polynomials $P_{m, j}(x)$ given by $P_{m, j}(x)=P_{j}(t)$ where $x$ and $t$ are related by (30). Obviously the polynomials $P_{m, j}$ are orthogonal with respect to the $L^{2}$ integral on $I_{m}$. We shall also make use of the following identities:

$$
\begin{aligned}
P_{j}( \pm 1) & =( \pm 1)^{j}, \quad j=0,1, \ldots, \\
P_{j}^{\prime}( \pm 1) & =\frac{1}{2}( \pm 1)^{j-1} j(j+1), \quad j=1, \ldots, \\
P_{j}^{\prime \prime}( \pm 1) & =\frac{1}{8}( \pm 1)^{j}(j-1) j(j+1)(j+2), \quad j=2, \ldots,
\end{aligned}
$$

which are easily proved by induction using well-known recursive relations of the Legendre polynomials. Writing $\left.\sigma\right|_{I_{m}}=\sum_{j=0}^{q+3} \alpha_{m, j} P_{m, j}(x)$ and using the last three equations of (23), in that order, we get

$$
\begin{aligned}
\sum_{j=0}^{q+3} \alpha_{m, j} P_{m, j}\left(x_{m}^{+}\right) & =\sum_{j=0}^{q+3} \alpha_{m, j} P_{j}(-1)=\sum_{j=0}^{q+3} \alpha_{m, j}(-1)^{j}=u\left(x_{m}^{-}\right) \\
\sum_{j=1}^{q+3} \alpha_{m, j} P_{m, j}^{\prime}\left(x_{m}^{+}\right) & =\frac{2}{h_{m}} \sum_{j=1}^{q+3} \alpha_{m, j} P_{j}^{\prime}(-1)=\frac{1}{h_{m}} \sum_{j=1}^{q+3} \alpha_{m, j}(-1)^{j-1} j(j+1)=\left\{u_{x}\right\}_{m} \\
\sum_{j=2}^{q+3} \alpha_{m, j} P_{m, j}^{\prime \prime}\left(x_{m}^{+}\right) & =\frac{4}{h_{m}^{2}} \sum_{j=2}^{q+3} \alpha_{m, j} P_{j}^{\prime \prime}(-1) \\
& =\frac{1}{2 h_{m}^{2}} \sum_{j=2}^{q+3} \alpha_{m, j}(-1)^{j}(j-1) j(j+1)(j+2)=u_{x x}\left(x_{m}^{+}\right) .
\end{aligned}
$$

Notice the upper triangular structure of the $3 \times(q+4)$ coefficient matrix. Obviously the diagonal elements of this matrix are nonzero. Next, from the first equation of (23) we get

$$
\sum_{j=3}^{q+3}\left(P_{m, j}^{\prime \prime \prime}, P_{m, i}\right)_{I_{m}} \alpha_{m, j}=\sum_{j=3+i}^{q+3}\left(P_{m, j}^{\prime \prime \prime}, P_{m, i}\right)_{I_{m}} \alpha_{m, j}=\left(\mathcal{D} u, P_{m, i}\right), \quad i=0, \ldots, q,
$$

using the fact that $P_{m, i}$ is orthogonal to all polynomials with degree less than $i$. Again notice the (shifted) upper triangular structure of the $(q+1) \times(q+4)$ coefficient matrix. Furthermore, the elements $\left(P_{m, 3+i}^{\prime \prime \prime}, P_{m, i}\right)_{I_{m}}$ of the matrix are nonzero. To see this, simply note that $\operatorname{deg} P_{m, 3+i}^{\prime \prime \prime}=i$ and $P_{m, i}$ is not orthogonal to $x^{i}$ whereas it is orthogonal to $x^{j}$ with $j<i$. Combining these equations with the previous three, we obtain a system whose matrix is upper triangular and invertible. Finally, notice 
that each row of the matrix is multiplied by some power of $h_{m}$. Upon transferring these terms to the right-hand side vector, the matrix can be rendered independent of the particular cell; thus it may be computed as a template and stored.

3.2. Approximation properties of the operator $\mathcal{R}$. We begin with a lemma that establishes a connection between the dispersive projection and reconstruction operators.

Lemma 3.1. Let $u \in H^{3}\left(\mathcal{T}_{h}\right)$ and suppose $\mathcal{P} u$ exists. Then,

$$
\mathcal{P} \mathcal{R} u=\mathcal{P} u .
$$

Proof. Again for convenience we use $\sigma=\mathcal{R} u$. Since $\sigma \in C^{2}[0,1] \cap H^{3}\left(\mathcal{T}_{h}\right)$, it follows from part (i) of Lemma 2.2 that $\left(\sigma_{x x x}, v\right)=(\mathcal{D} \sigma, v), \forall v \in V_{h}^{q}$. Hence, in view of (12) we obtain

$$
\begin{aligned}
\left(\sigma_{x x x}, v\right)= & -\sum_{I \in \mathcal{T}_{h}}\left(\sigma, v_{x x x}\right)_{I}-\sum_{m=0}^{M-1} \sigma_{x x}^{+}[v]_{m}+\sum_{m=0}^{M-1}\left\{\sigma_{x}\right\}_{m}\left[v_{x}\right]_{m} \\
& -\sum_{m=0}^{M-1} \sigma_{m}^{-}\left[v_{x x}\right]_{m}, \quad \forall v \in V_{h}^{q} .
\end{aligned}
$$

Now by the definition of $\sigma$ we have $\left(\sigma_{x x x}, v\right)=(\mathcal{D} u, v), \forall v \in V_{h}^{q}$ and $\left\{\sigma_{x}\right\}_{m}=$ $\sigma_{x}\left(x_{m}^{+}\right)$and $\sigma_{m}^{-}=\sigma_{m}^{+}$in view of its smoothness. Hence, comparing the above equation with $(\mathcal{D} u, v)$ as given by (12), we see that $\left(\sigma, v_{x x x}\right)_{I}=\left(u, v_{x x x}\right)_{I}, \forall v \in$ $V_{h}^{q}(I)$ or equivalently $(\sigma, v)_{I}=(u, v)_{I}, \forall v \in V_{h}^{q-3}(I)$. Finally, using (20) and (23) together with the fact that $\left\{\sigma_{x}\right\}_{m}=\sigma_{x}\left(x_{m}^{+}\right)$and $\sigma_{m}^{-}=\sigma_{m}^{+}$we see that for all cells $I=\left[x_{m}, x_{m+1}\right], m=0, \ldots, M-1$ there holds

$$
\begin{aligned}
(\sigma, v)_{I} & =(u, v)_{I}=(\mathcal{P} u, v)_{I}, \quad \forall v \in V_{h}^{q-3}(I) \\
\sigma\left(x_{m}^{-}\right) & =u\left(x_{m}^{-}\right)=(\mathcal{P} u)\left(x_{m}^{-}\right) \\
\left\{\sigma_{x}\right\}_{m} & =\left\{u_{x}\right\}_{m}=\left\{(\mathcal{P} u)_{x}\right\}_{m}, \\
\sigma_{x x}\left(x_{m}^{+}\right) & =u_{x x}\left(x_{m}^{+}\right)=(\mathcal{P} u)_{x x}\left(x_{m}^{+}\right) .
\end{aligned}
$$

The assertion of the lemma follows at once.

The next results will prove useful in the context of the error indicator $\mathcal{E}_{2}$ given by (53). The first estimate asserts that when $u$ is appropriately smooth, $u$ and $\mathcal{R} u$ are optimally close. In the second estimate, the smoothness assumption is relaxed though it is still possible to bound $u-\mathcal{R} u$ in various norms by including jump terms. In fact, it may also be profitably applied when $u \in V_{h}^{q}$ as indicated in Remark 3.2 below.

Theorem 3.2. (i) Suppose $u \in C^{2}[0,1] \cap H^{q+4}\left(\mathcal{T}_{h}\right)$. Then, for each $I \in \mathcal{T}_{h}$,

$$
|u-\mathcal{R} u|_{j, I} \leq c h_{I}^{q+4-j}|u|_{q+4, I}, \quad j=0, \ldots, q+4 .
$$

(ii) Suppose $u \in H^{q+4}\left(\mathcal{T}_{h}\right)$. Then, for $j=0, \ldots, 3$, and $I=\left[x_{m}, x_{m+1}\right] \in \mathcal{T}_{h}$,

$$
\begin{aligned}
|u-\mathcal{R} u|_{j, I} \leq c\left(h_{I}^{q+4-j}|u|_{q+4, I}+h_{m}^{\frac{1}{2}-j}\left|[u]_{m}\right|+h_{m}^{\frac{3}{2}-j}\left(\left|\left[u_{x}\right]_{m}\right|+\left|\left[u_{x}\right]_{m+1}\right|\right)\right. \\
\left.+h_{m}^{\frac{5}{2}-j}\left|\left[u_{x x}\right]_{m+1}\right|\right) .
\end{aligned}
$$


Proof. (i) We observed in Remark 3.1 that $\sigma_{x x x}=\mathcal{D} u$ and in part (ii) of Remark 2.1 that if $u$ belongs to $C^{2}[0,1]$ then $\left.\mathcal{D} u\right|_{I}$ is the $L^{2}$ projection of $\left.u_{x x x}\right|_{I}$ into $V_{h}^{q}(I)$. In view of standard approximation theory we have

$$
\left|\sigma_{x x x}-u_{x x x}\right|_{j, I} \leq c h_{I}^{q+1-j}\left|u_{x x x}\right|_{q+1, I}=c h_{I}^{q+1-j}|u|_{q+4, I}, \quad j=0, \ldots, q+1,
$$

which shows that (34) holds for $j=3, \ldots, q+4$. Now with $I=\left[x_{m}, x_{m+1}\right]$, since $\sigma_{x x}\left(x_{m}^{+}\right)=u_{x x}\left(x_{m}^{+}\right)$, we have for $x \in I$,

$$
\begin{aligned}
\left|\sigma_{x x}(x)-u_{x x}(x)\right|= & \left|\int_{x_{m}}^{x}\left(\sigma_{x x x}(t)-u_{x x x}(t)\right) d t\right| \\
& \leq \operatorname{ch}_{I}^{1 / 2}\left\|\sigma_{x x x}-u_{x x x}\right\|_{I} \leq c h_{I}^{q+3 / 2}|u|_{q+4, I},
\end{aligned}
$$

using (36) with $j=0$ in the last step. From this, it easily follows upon integration that

$$
\left\|\sigma_{x x}-u_{x x}\right\|_{I} \leq c h_{I}^{q+2}|u|_{q+4, I},
$$

establishing (34) for $j=2$. Similar arguments, using the facts that $\sigma_{x}\left(x_{m}^{+}\right)=$ $\left\{u_{x}\right\}_{m}=u_{x}\left(x_{m}^{+}\right)$and $\sigma\left(x_{m}^{-}\right)=u\left(x_{m}^{-}\right)$can be used to handle the cases $j=0,1$.

(ii) Unlike the situation in part (i), it is no longer the case that $\left.\sigma_{x x x}\right|_{I}$ is the $L^{2}$ projection of $\left.u_{x x x}\right|_{I}$ in view of the jumps of $u$. In order to handle this difficulty, we let $\eta$ denote the $L^{2}$ projection of $u_{x x x}$ into $V_{h}^{q}$. Proceeding as in Lemma 2.3 and using the fact that $\sigma_{x x x}=\mathcal{D} u$, we obtain

$$
\left(\sigma_{x x x}-\eta, v\right)=\sum_{I \in \mathcal{T}_{h}}\left(u_{x x x}-\eta, v\right)_{I}+\sum_{j=0}^{M-1}\left[u_{x x}\right]_{j} v_{j}^{-}-\sum_{j=0}^{M-1}\left[u_{x}\right]_{j}\left\{v_{x}\right\}_{j}+\sum_{j=0}^{M-1}[u]_{j} v_{x x}\left(x_{j}^{+}\right) .
$$

Now letting $\eta_{I}=\eta \chi_{I}$ and $v=\left(\sigma_{x x x}-\eta\right) \chi_{I}$ and following the same steps as in the proof of Lemma 2.3, we obtain

$$
\begin{array}{r}
\left\|\sigma_{x x x}-\eta_{I}\right\|_{I}^{2} \leq c\left(\left\|u_{x x x}-\eta_{I}\right\|_{I}^{2}+h_{m}^{-5}[u]_{m}^{2}+h_{m}^{-3}\left(\left[u_{x}\right]_{m}^{2}+\left[u_{x}\right]_{m+1}^{2}\right)\right. \\
\left.+h_{m}^{-1}\left[u_{x x}\right]_{m+1}^{2}\right) .
\end{array}
$$

In view of the approximation properties of $\eta_{I}$ we have

$$
\left\|u_{x x x}-\eta_{I}\right\|_{I} \leq c h_{m}^{q+1}\left|u_{x x x}\right|_{q+1, I}=c h_{m}^{q+1}|u|_{q+4, I} .
$$

It then follows from (37) and the triangle inequality that

$$
\left\|\sigma_{x x x}-u_{x x x}\right\|_{I}^{2} \leq c\left(h_{m}^{q+1}|u|_{q+4, I}^{2}+h_{m}^{-5}[u]_{m}^{2}+h_{m}^{-3}\left(\left[u_{x}\right]_{m}^{2}+\left[u_{x}\right]_{m+1}^{2}\right)+h_{m}^{-1}\left[u_{x x}\right]_{m+1}^{2}\right) .
$$

This bound constitutes the essential part of the proof and yields (35) for $j=3$. The case $j=2$ can now be handled by using the The Fundamental Theorem of Calculus as was done in the proof of part (i) above. For $j=1$, there is a minor technical detail to be taken care of. Indeed, we write, in view of the third equation of (23)

$$
\sigma_{x}(x)-u_{x}(x)=-\frac{1}{2}\left[u_{x}\right]_{m}+\int_{x_{m}}^{x}\left(\sigma_{x x}-u_{x x}\right)(t) d t,
$$

from which it follows as in part (i) that

$$
\left\|\sigma_{x}-u_{x}\right\|_{I} \leq c h_{m}^{1 / 2}\left|\left[u_{x}\right]_{m}\right|+h_{m}\left\|\sigma_{x x}-u_{x x}\right\|_{I} .
$$


Using this and the fact that (35) has already been shown to hold for $j=2$, establishes it for $j=1$. The remaining case $j=0$ can be established in a similar manner. This concludes the proof.

Remark 3.2. Theorem 3.2 can be applied, in particular, to $u=v_{h} \in V_{h}^{q}$ to yield

$\left|v_{h}-\mathcal{R} v_{h}\right|_{j, I} \leq c\left(h_{m}^{\frac{1}{2}-j}\left|\left[v_{h}\right]_{m}\right|+h_{m}^{\frac{3}{2}-j}\left(\left|\left[v_{h, x}\right]_{m}\right|+\left|\left[v_{h, x}\right]_{m+1}\right|\right)+h_{m}^{\frac{5}{2}-j}\left|\left[v_{h, x x}\right]_{m+1}\right|\right)$.

To put this result in perspective, suppose $v_{h} \in V_{h}^{q}$ is an optimal approximation to a smooth function $u$ (e.g., the solution of the GKdV equation (10) in the sense that

$$
\left\|u-v_{h}\right\|_{I}+h_{I}\left\|u_{x}-v_{h x}\right\|_{I} \leq c h_{I}^{q+1}, \forall I \in \mathcal{T}_{h} .
$$

Using the fact that the jumps of $u$ and its derivatives vanish at the nodes, it follows from the embedding inequality (3) that $\left|v_{h}-\mathcal{R} v_{h}\right|_{j, I}=O\left(h_{I}^{q+1-j}\right)$.

\section{A POSTERIORI ERROR ESTIMATES FOR THE SEMIDISCRETE APPROXIMATION}

In 11 we defined the semidiscrete approximation to the solution $u$ of (11) as the function $u_{h}:[0, T] \rightarrow V_{h}^{q}$ given by

$$
u_{h t}+\mathcal{N}\left(u_{h}\right)+\epsilon \mathcal{D} u_{h}=0, \quad t \in(0, T], \quad u_{h}(0)=P u(0)
$$

with $P$ representing an appropriate operator into $V_{h}^{q}$ possessing good approximation properties, e.g., $\|u-P u\|=O\left(h^{q}\right)$. Indeed, the $L^{2}$ projection into $V_{h}^{q}$ or the Lagrange interpolant satisfy this requirement.

In [1] the following a priori bound for the semidiscrete scheme (40) was established:

$$
\left\|\left(u-u_{h}\right)(t)\right\| \leq \operatorname{ch}^{q}, \quad 0 \leq t \leq T .
$$

The next result shows how a posteriori error control of $u-u_{h}$ is obtained in terms of the error $u_{h}-\mathcal{R} u_{h}$. Notice that since $\mathcal{R} u_{h}$ can be explicitly constructed from $u_{h}$, the terms on the right-hand side of the estimate in Theorem 4.1 are computable. This of course, relies on the assumption that $u_{h}$ has been computed by some means, e.g., the Method of Lines.

Theorem 4.1. Let $u_{h}$ be the solution of the scheme (40) and let $\sigma=\mathcal{R} u_{h}$ be the dispersive reconstruction of $u_{h}$ as defined in Theorem 3.1. Then for $t \geq 0$,

$$
\begin{gathered}
\left\|\left(u-u_{h}\right)(t)\right\| \leq e^{c t}\left(\|(u-\sigma)(0)\|+\left\|\left(u_{h}-\sigma\right)(t)\right\|+\int_{0}^{t}\left(\left\|\left(u_{h t}-\sigma_{t}\right)(t)\right\|\right.\right. \\
\left.\left.+\left\|\mathcal{N}\left(u_{h}\right)-\left(\sigma^{p+1}\right)_{x}\right\|\right)\right),
\end{gathered}
$$

where the constant $c$ depends on $\|u\|_{W^{1, \infty}}$ and $\|\sigma\|_{W^{1, \infty}}$ for $p \geq 2$ and only on $\left\|u_{x}\right\|_{\infty}$ for $p=1$.

Proof. Recall that $\sigma$ belongs to $C^{2}[0,1] \cap V_{h}^{q+3}$ and that $\sigma_{x x x}=\mathcal{D} u_{h}$. Therefore from the semidiscrete equation we have $u_{h t}+\mathcal{N}\left(u_{h}\right)+\epsilon \sigma_{x x x}=0$.

Letting $e=u-\sigma$ where $u$ is the solution of (1) we get

$$
e_{t}+\left(u^{p+1}\right)_{x}-\left(\sigma^{p+1}\right)_{x}+\epsilon e_{x x x}=u_{h t}-\sigma_{t}+\mathcal{N}\left(u_{h}\right)-\left(\sigma^{p+1}\right)_{x} .
$$


Multiplying with $e$ and integrating, we have

$$
\frac{1}{2} \frac{d}{d t}\|e\|^{2}+\frac{1}{2}\left(\left(\sum_{j=0}^{p} u^{j} \sigma^{p-j}\right)_{x}, e^{2}\right)=\left(u_{h t}-\sigma_{t}+\mathcal{N}\left(u_{h}\right)-\left(\sigma^{p+1}\right)_{x}, e\right) .
$$

Hence, from Gronwall's inequality, it follows that

$$
\|e(t)\| \leq e^{c t}\left(\|e(0)\|+\int_{0}^{t}\left(\left\|u_{h t}-\sigma_{t}+\mathcal{N}\left(u_{h}\right)-\left(\sigma^{p+1}\right)_{x}\right\|\right)\right)
$$

where the constant $c$ depends on $\|u\|_{W^{1, \infty}}$ and $\|\sigma\|_{W^{1, \infty}}$. Note, however, that in the case $p=1$,

$$
\left(\left(\sum_{j=0}^{p} u^{j} \sigma^{p-j}\right)_{x}, e^{2}\right)=\left(2 u_{x}, e^{2}\right)-\left(e_{x}, e^{2}\right)=2\left(u_{x}, e^{2}\right),
$$

since the term $\left(e_{x}, e^{2}\right)=0$ by periodicity. Hence the constant $c$ does not depend on $\sigma$ in this case. The conclusion of the theorem now follows from the triangle inequality.

The following remarks are in order:

Remark 4.1. Due to the presence of the nonlinear term in the GKdV equation the term $\left\|\mathcal{N}\left(u_{h}\right)-\left(\sigma^{p+1}\right)_{x}\right\|$ is present in the estimate (42). Notice that this estimator is expected to be of $O\left(h^{q}\right)$ due to the differentiation with respect to $x$. A fact which is consistent with the a priori results [11,13. In addition, in the above theorem we did not insist on a more careful treatment of the nonlinearity as far as conditional estimators are concerned. Indeed, it is possible in certain nonlinear equations to show estimates under assumptions based solely on the approximate solution. These estimates are based on subtle PDE arguments (see e.g. [23] and in particular [19] treating the case of a nonlinear parabolic equation with possible finite time blow-up) and are beyond the scope of the present paper.

Remark 4.2. In the case of the linear dispersive equation

$$
u_{t}+u_{x x x}=0
$$

one can prove the estimate

$$
\left\|\left(u-u_{h}\right)(t)\right\| \leq C\left(\left\|\left(u_{h t}-\sigma_{t}\right)(t)\right\|+\left\|\left(u_{h}-\sigma\right)(t)\right\|+\|(u-\sigma)(0)\|\right)
$$

where $C$ is a constant independent of $u$ and $u_{h}$. The above bound is an $O\left(h^{q+1}\right)$ estimator of the error.

\section{A POSTERIORI ERROR ESTIMATES FOR A FUlLy DisCRETE SCHEME}

The approach we will follow in deriving a posteriori error estimates for fully discrete approximations is to form a pair of two time-stepping schemes. The first is used to generate the fully discrete approximations and the second to supply the estimation.

The difficulty here resides mainly in the fact that fully discrete approximations are indeed discrete whereby there is a need for a function which is continuous in time and satisfies the same differential equation as (11) with a computable right-hand side. 
5.1. The Implicit Euler method. Let $0 \leq t^{0}<t^{1}<\cdots<t^{N}=T$ be a partition of the interval $[0, T]$ and $\kappa_{n}=t^{n+1}-t^{n}$. The fully discrete approximations $u^{n}$ to $u\left(\cdot, t^{n}\right)$ generated by the Implicit Euler method are given by

$$
u^{n+1}-u^{n}+\kappa_{n} \mathcal{N}\left(u^{n+1}\right)+\kappa_{n} \epsilon \mathcal{D} u^{n+1}=0, \quad n=0, \ldots, N-1,
$$

with $u^{0}:=u_{h}(0)$.

In order to estimate the errors of the Implicit Euler method we will use, at every step, the midpoint rule which is given by

$$
u_{M}^{n+1}-u^{n}+\kappa_{n} \mathcal{N}\left(u_{M}^{n, 1}\right)+\kappa_{n} \epsilon \mathcal{D} u_{M}^{n, 1}=0, \quad \text { where } \quad u_{M}^{n, 1}=\left(u_{M}^{n+1}+u^{n}\right) / 2 .
$$

Note that we are using the same value $u^{n}$ generated by the Implicit Euler method as initial value for the midpoint rule and we are using the subscript $M$ for the approximations generated by the midpoint rule.

That both of these schemes are well defined can be established by using a variant of Brouwer's fixed point Theorem (cf. [5]). Uniqueness and convergence can be proved under appropriate CFL type conditions.

In addition, the convergence rates

$$
\left\|u\left(\cdot, t^{n}\right)-u^{n}\right\|=O\left(h^{q}+\kappa\right), \quad \kappa=\max _{0 \leq n \leq N} \kappa_{n}
$$

can be obtained; for details see [5, 15].

To derive a posteriori estimates for these schemes we combine ideas of [3, 7 ] and of the semidiscrete case considered previously. Notice first that we make the simplifying assumption that the finite element spaces do not change with time. The general case can be treated also along the lines of [7] but we do not insist on this in the present paper.

The fully discrete reconstruction is defined as the function $\hat{U}:[0, T] \rightarrow C^{2}[0,1] \cap$ $V_{h}^{q+3}$ which on each interval $I_{n}=\left[t^{n}, t^{n+1}\right]$ is given by

$$
\hat{U}(t)=\mathcal{R}\left[u^{n}+\int_{t^{n}}^{t} F(s) d s\right],
$$

here $F(\cdot)$ is the affine in $t$ function given by

$$
F(t)=-\ell_{1 / 2}(t)\left\{\mathcal{N}\left(u_{M}^{n, 1}\right)+\epsilon \mathcal{D} u_{M}^{n, 1}\right\}-\ell_{1}(t)\left\{\mathcal{N}\left(u^{n+1}\right)+\epsilon \mathcal{D} u^{n+1}\right\},
$$

where $\ell_{1 / 2}(t)$ and $\ell_{1}(t)$ are the two basis functions of the space of affine functions in $t$ on $I_{n}$ corresponding to the nodes $t^{n, 1}:=\left(t^{n}+t^{n+1}\right) / 2$ and $t^{n+1}$, respectively. More specifically,

$$
\ell_{1 / 2}(t)=-\frac{2}{\kappa_{n}}\left(t-t^{n+1}\right), \quad \ell_{1}(t)=\frac{2}{\kappa_{n}}\left(t-t^{n, 1}\right) .
$$

Notice that $\hat{U}$ is a computable piecewise polynomial function. Furthermore, the next lemma shows that it is related to the function $U(t)=\left(\left(t^{n+1}-t\right) u^{n}+(t-\right.$ $\left.\left.t^{n}\right) u^{n+1}\right) / \kappa_{n}$, i.e., the affine interpolant of the nodal values $u^{n}$ and $u^{n+1}$.

Lemma 5.1. Let $U(t)$ be given as above. Then

$$
\hat{U}(t)=\mathcal{R}\left\{U(t)+\left[\frac{3}{4} \hat{\ell}_{1 / 2}(t)+\hat{\ell}_{1}(t)\right]\left(u_{M}^{n+1}-u^{n+1}\right)\right\}, \quad t^{n} \leq t \leq t^{n+1},
$$

where the quadratic functions $\hat{\ell}_{1 / 2}(t)$ and $\hat{\ell}_{1}(t)$ are given by

$$
\hat{\ell}_{1 / 2}(t)=-\frac{4}{\kappa_{n}^{2}}\left(t-t^{n}\right)\left(t-t^{n+1}\right), \quad \hat{\ell}_{1}(t)=\frac{2}{\kappa_{n}^{2}}\left(t-t^{n}\right)\left(t-t^{n, 1}\right) .
$$


Proof. Since $F(t)$ is affine, and the midpoint rule of quadrature is exact for such functions, from (46), (47) and (45) we obtain

$$
\begin{aligned}
\hat{U}\left(t^{n+1}\right) & =\mathcal{R}\left\{u^{n}+\int_{t^{n}}^{t^{n+1}} F(s) d s\right\}=\mathcal{R}\left\{u^{n}+\kappa_{n} F\left(t^{n, 1}\right)\right\} \\
& =\mathcal{R}\left\{u^{n}-\kappa_{n}\left(\mathcal{N}\left(u_{M}^{n, 1}\right)+\epsilon \mathcal{D} u_{M}^{n, 1}\right)\right\}=\mathcal{R} u_{M}^{n+1} \\
& =\mathcal{R}\left\{u^{n+1}\right\}+\mathcal{R}\left\{u_{M}^{n+1}-u^{n+1}\right\} .
\end{aligned}
$$

Also, since $\ell_{1 / 2}\left(t^{n}\right)=2, \ell_{1}\left(t^{n}\right)=-1, \ell_{1 / 2}\left(t^{n, 1}\right)=1, \ell_{1}\left(t^{n, 1}\right)=0$ and the trapezoidal rule is exact for affine functions, we obtain from (45) and (44)

$$
\begin{aligned}
\hat{U}\left(t^{n, 1}\right) & =\mathcal{R}\left\{u^{n}+\frac{\kappa_{n}}{4}\left[F\left(t^{n}\right)+F\left(t^{n, 1}\right)\right]\right\} \\
& =\mathcal{R}\left\{u^{n}-\frac{\kappa_{n}}{4}\left[3\left(\mathcal{N}\left(u_{M}^{n, 1}\right)+\epsilon \mathcal{D} u_{M}^{n, 1}\right)-\left(\mathcal{N}\left(u^{n+1}\right)+\epsilon \mathcal{D} u^{n+1}\right)\right]\right\} \\
(50) \quad & \mathcal{R}\left\{\frac{1}{2} u^{n}+\frac{3}{4} u_{M}^{n+1}-\frac{1}{4} u^{n+1}\right\}=\mathcal{R}\left\{\frac{1}{2}\left(u^{n}+u^{n+1}\right)+\frac{3}{4}\left(u_{M}^{n+1}-u^{n+1}\right)\right\} \\
& =\mathcal{R}\left\{U\left(t^{n, 1}\right)+\frac{3}{4}\left(u_{M}^{n+1}-u^{n+1}\right)\right\} .
\end{aligned}
$$

Finally, since $\hat{U}\left(t^{n}\right)=\mathcal{R} u^{n}=\mathcal{R} U\left(t^{n}\right)$, the result (48) follows from (49) and (50) and the fact that $\tilde{\ell}_{1 / 2}(t)$ and $\tilde{\ell}_{1}(t)$ are the Lagrange basis functions corresponding to the points $t^{n, 1}$ and $t^{n+1}$, respectively.

We next derive an error equation for $\rho(t):=\hat{U}(t)-u(t)$.

Lemma 5.2. $\rho(t)$ satisfies

$$
\rho_{t}+\left(\hat{U}^{p+1}\right)_{x}-\left(u^{p+1}\right)_{x}+\epsilon \rho_{x x x}=\mathcal{E}_{1}+\mathcal{E}_{2}+\mathcal{E}_{3}
$$

where the error indicators $\mathcal{E}_{1}, \mathcal{E}_{2}, \mathcal{E}_{3}$ are given by

$$
\begin{aligned}
\mathcal{E}_{1} & =\left(\hat{U}^{p+1}\right)_{x}-\mathcal{R}\left\{\ell_{1 / 2}(t) \mathcal{N}\left(u_{M}^{n, 1}\right)+\ell_{1}(t) \mathcal{N}\left(u^{n+1}\right)\right\} \\
\mathcal{E}_{2} & =\epsilon(I-\mathcal{R}) \mathcal{D}\left(U(t)+\frac{1}{2} \ell_{1 / 2}(t)\left(u_{M}^{n+1}-u^{n+1}\right)\right) \\
\mathcal{E}_{3} & =\epsilon\left[\frac{3}{4} \hat{\ell}_{1 / 2}(t)+\hat{\ell}_{1}(t)-\frac{1}{2} \ell_{1 / 2}(t)\right] \mathcal{D}\left(u_{M}^{n+1}-u^{n+1}\right) .
\end{aligned}
$$

Proof. From the definitions of $F(t)$ and $U(t)$ we have

$$
\begin{gathered}
\hat{U}_{t}=\mathcal{R} F(t)=-\mathcal{R}\left\{\ell_{1 / 2}(t)\left(\mathcal{N}\left(u_{M}^{n, 1}\right)+\epsilon \mathcal{D} u_{M}^{n, 1}\right)+\ell_{1}(t)\left(\mathcal{N}\left(u^{n+1}\right)+\epsilon \mathcal{D} u^{n+1}\right)\right\} \\
(55)=-\mathcal{R}\left\{\ell_{1 / 2}(t) \mathcal{N}\left(u_{M}^{n, 1}\right)+\ell_{1}(t) \mathcal{N}\left(u^{n+1}\right)+\epsilon \mathcal{D} U(t)\right. \\
\left.+\frac{1}{2} \epsilon \ell_{1 / 2}(t) \mathcal{D}\left(u_{M}^{n+1}-u^{n+1}\right)\right\}
\end{gathered}
$$

where we have used the linearity of the operator $\mathcal{D}$. On the other hand, from (48) it follows that

$$
\hat{U}_{x x x}(t)=\mathcal{D} U(t)+\left[\frac{3}{4} \hat{\ell}_{1 / 2}(t)+\hat{\ell}_{1}(t)\right] \mathcal{D}\left(u_{M}^{n+1}-u^{n+1}\right) .
$$


Combining (55) and (56), adding $\left(\hat{U}^{p+1}\right)_{x}$ to both sides and using (11) we obtain (51).

The next result provides the a posteriori estimate for the fully discrete scheme generated by the Implicit Euler scheme (44). In doing so we also define the error indicator

$$
\mathcal{E}_{4}^{n}=\frac{1}{\sqrt{\kappa_{n}}}\left(\hat{U}\left(t^{n}\right)-\hat{U}\left(t^{n-}\right)\right)=\frac{1}{\sqrt{\kappa_{n}}}\left(\mathcal{R}\left(u_{M}^{n}-u^{n}\right)\right), \quad n=1,2, \ldots,
$$

which makes its appearance due to the fact that the function $\hat{U}(t)$ is discontinuous at the temporal nodes $t^{1}, \ldots, t^{N-1}$.

Theorem 5.1. Let $u^{n}$ be the solution of the fully discrete scheme (44), and let $\hat{U}$ the discrete reconstruction defined by (46). With the error indicators $\mathcal{E}_{1}, \mathcal{E}_{2}, \mathcal{E}_{3}, \mathcal{E}_{4}$ given by (52), (53), (54) and (57), there holds the a posteriori error estimate

$$
\left\|u\left(t^{n}\right)-u^{n}\right\| \leq\left\|u^{n}-\hat{U}\left(t^{n}\right)\right\|+c e^{c t^{n}}\left(\left\|u^{0}-u^{0}\right\|^{2}+\sum_{i=1}^{3} \int_{0}^{t^{n}}\left\|\mathcal{E}_{i}(s)\right\|^{2} d s+\sum_{j=1}^{n-1}\left\|\mathcal{E}_{4}^{j}\right\|^{2}\right)^{1 / 2} .
$$

Proof. Letting $\mathcal{E}:=\mathcal{E}_{1}+\mathcal{E}_{2}+\mathcal{E}_{3}$, multiplying both sides of (51) by $\rho$ and integrating with respect to $x$ gives

$$
\frac{1}{2} \frac{d}{d t}\|\rho(t)\|^{2}+\left(\left(\hat{U}^{p+1}\right)_{x}-\left(u^{p+1}\right)_{x}, \rho(t)\right)=(\mathcal{E}(t), \rho(t)), \quad t^{n} \leq t \leq t^{n+1} .
$$

We would like to use Gronwall's Lemma. However, we have to deal with the complication arising from the fact that $\hat{U}$ and thus $\rho$ has jumps at $t^{1}, \ldots, t^{N-1}$. Now as done in the semidicrete case, we have the bound

$$
\left|\left(\left(\hat{U}^{p+1}\right)_{x}-\left(u^{p+1}\right)_{x}, \rho(t)\right)\right|=\frac{1}{2}\left|\sum_{j=0}^{p}\left(\left(u^{j} \rho^{p-j}\right)_{x}, \rho^{2}\right)\right| \leq c\|\rho(t)\|^{2},
$$

where $c$ depends on the $\max _{t^{n} \leq t \leq t^{n+1}}\|u(t)\|_{1, \infty}$ and $\max _{t^{n} \leq t \leq t^{n+1}}\|\hat{U}(t)\|_{1, \infty}$. Thus integrating (59) from $t^{n}$ to $t \in\left[t^{n}, t^{n+1}\right]$ and using the arithmetic-geometric mean inequality, we obtain

$$
\|\rho(t)\|^{2} \leq\left\|\rho\left(t^{n}\right)\right\|^{2}+c \int_{t^{n}}^{t}\|\rho(s)\|^{2} d s+c \int_{t^{n}}^{t}\|\mathcal{E}(s)\|^{2} d s .
$$

From the mean value theorem for integrals we obtain,

$$
\max _{t^{n} \leq t \leq t^{n+1}}\|\rho(t)\|^{2} \leq\left(1+c \kappa_{n}\right)\left(\left\|\rho\left(t^{n}\right)\right\|^{2}+c \int_{t^{n}}^{t^{n+1}}\|\mathcal{E}(s)\|^{2} d s\right) .
$$

In particular, we have

$$
\left\|\rho\left(t^{n+1-}\right)\right\|^{2} \leq\left(1+c \kappa_{n}\right)\left(\left\|\rho\left(t^{n}\right)\right\|^{2}+c \int_{t^{n}}^{t^{n+1}}\|\mathcal{E}(s)\|^{2} d s\right) .
$$

Now, since $u$ is a smooth function of $t$, we have

$$
\left\|\rho\left(t^{n}\right)\right\|^{2}-\left\|\rho\left(t^{n-}\right)\right\|^{2}=\left(\hat{U}\left(t^{n}\right)-\hat{U}\left(t^{n-}\right), \rho\left(t^{n}\right)+\rho\left(t^{n-}\right)\right),
$$

from which we easily obtain

$$
\left\|\rho\left(t^{n}\right)\right\|^{2} \leq\left(1+c \kappa_{n}\right)\left(\left\|\rho\left(t^{n-}\right)\right\|^{2}+\frac{1}{\kappa_{n}}\left\|\hat{U}\left(t^{n}\right)-\hat{U}\left(t^{n-}\right)\right\|^{2}\right) .
$$


Using (65) in (63) and a discrete version of Gronwall's Lemma, we obtain

$$
\max _{0 \leq t \leq T}\|\rho(t)\|^{2} \leq c e^{c T}\left(\|\rho(0)\|^{2}+\sum_{n=1}^{N-1} \frac{1}{\kappa_{n}}\left\|\hat{U}\left(t^{n}\right)-\hat{U}\left(t^{n-}\right)\right\|^{2}+\int_{0}^{T}\|\mathcal{E}(s)\|^{2} d s\right) .
$$

The conclusion now follows from the triangle inequality and the observation that $\hat{U}\left(t^{n}\right)=\mathcal{R} u^{n}$.

\section{A posteriori error estimates for the Cheng-Shu Formulation}

In this section we show that the machinery developed in this paper can be adapted successfully to the method developed in [13. More specifically, we will show that analogs of all the results contained herein can be duplicated for their method. Let us recall that the approach followed in 13 consisted in using dissipative versions $\tilde{\mathcal{N}}$ and $\tilde{\mathcal{D}}$ of the forms $\mathcal{N}$ and $\mathcal{D}$. Since the form $\tilde{\mathcal{D}}$ will play a central role, we recall its definition:

$$
\tilde{\mathcal{D}}(u, v)=-\sum_{I \in \mathcal{T}_{h}}\left(u, v_{x x x}\right)_{I}-\sum_{j=0}^{M-1} u_{x x}\left(x_{j}^{+}\right)[v]_{j}+\sum_{j=0}^{M-1} u_{x}\left(x_{j}^{+}\right)\left[v_{x}\right]_{j}-\sum_{j=0}^{M-1} u_{j}^{-}\left[v_{x x}\right]_{j} .
$$

The associated operator $\tilde{\mathcal{D}}: H^{3}\left(\mathcal{T}_{h}\right) \rightarrow V_{h}^{q}$ can be shown to satisfy the bound (15) of Lemma 2.3 using exactly the same proof. Furthermore, the corresponding reconstruction operator $\tilde{\mathcal{R}}$ can be constructed exactly along the lines of Theorem 3.1

Theorem 6.1. For each $u \in H^{3}\left(\mathcal{T}_{h}\right)$ there corresponds a unique $\tilde{\sigma}=\tilde{\mathcal{R}} u \in$ $C^{2}[0,1] \cap V_{h}^{q+3}$ such that for each cell $I=\left[x_{m}, x_{m+1}\right], \quad m=0, \ldots, M-1$ in $\mathcal{T}_{h}$ there holds

$$
\begin{aligned}
\left(\tilde{\sigma}_{x x x}, v\right)_{I} & =(\tilde{\mathcal{D}} u, v) \quad \forall v \in V_{h}^{q}(I), \\
\tilde{\sigma}\left(x_{m}^{+}\right) & =u\left(x_{m}^{-}\right), \\
\tilde{\sigma}_{x}\left(x_{m}^{+}\right) & =u_{x}\left(x_{m}^{+}\right) \\
\tilde{\sigma}_{x x}\left(x_{m}^{+}\right) & =u_{x x}\left(x_{m}^{+}\right) .
\end{aligned}
$$

The proof is exactly the same as that of Theorem 3.1. Furthermore, the calculation of $\tilde{\mathcal{R}} u$ is similar to that of $\mathcal{R} u$ whereby the coefficient matrices are the same but the right-hand side vectors are different.

Lemma 3.1 remains valid in the form $\tilde{\mathcal{P}} \tilde{\mathcal{R}} u=\tilde{\mathcal{P}} u$ where $\tilde{\mathcal{P}}$ is the dispersive projection operator introduced in [13] and which differs from $\mathcal{P}$ only in the third equation of (20) whereby $(\tilde{\mathcal{P}} u)_{x}\left(x_{m}^{+}\right)=u_{x}\left(x_{m}^{+}\right)$. An approximation result analogous to that of Theorem 3.2 also remains valid for the operator $\tilde{\mathcal{R}}$.

Fully discrete scheme analogs to (44) and (45) for the method of Cheng-Shu can be defined by simply replacing the operators $\mathcal{N}$ and $\mathcal{D}$ by $\tilde{\mathcal{N}}$ and $\tilde{\mathcal{D}}$, respectively. Furthermore, using the reconstruction $\tilde{\mathcal{R}}$ instead of $\mathcal{R}$ the analog of the fully discrete reconstruction $\hat{U}$ can be defined as well. Following these changes, all the results of $\S 5$ remain valid for their method as well.

\section{NUMERICAL EXPERIMENTS}

Numerical experiments designed to gauge the performance of our a posteriori error estimates are reported in this section. Interest is given particularly to two issues: 
(1) Validation of the theoretical results, including a study of the effectiveness error indicators $\mathcal{E}_{1}, \mathcal{E}_{2}, \mathcal{E}_{3}, \mathcal{E}_{4}$ individually and in combination as a gauge of the error $\left\|u\left(t^{n}\right)-u^{n}\right\|$.

(2) The experimental confirmation that the a posteriori upper bound obtained (right-hand side of (58) ) decreases at the same $O(\kappa)$ rate of the underlying fully discrete method. In that sense, these estimates can be qualified as being of optimal temporal order of accuracy.

In the numerical experiments reported here we used the classical solitary-waves

$$
u(x, t)=A \operatorname{sech}^{2 / p}\left(K\left(x-\omega t-x_{0}\right)\right),
$$

which are solutions of the Cauchy problem for (1) for any positive value of the amplitude $A$ provided $K$ and $\omega$ are given by

$$
K=\frac{p A^{p / 2}}{2 \epsilon(p+1)(p+2)}, \quad \omega=\frac{2 A^{p}}{(p+1)(p+2)} .
$$

Of course, the solitary waves are not periodic in space, but owing to the exponential decay of the hyperbolic secant function, they can be treated as periodic by simply restricting them to the computational domain $[0,1]$ and imposing periodic boundary conditions across $x=0$ and $x=1$. Also, we choose $x_{0}=.5$ so that the waves commence their evolution centered in the period domain.

As alternatives to the solitary waves, we also use the so-called cnoidal-wave solutions

$$
u(x, t)=a \mathrm{cn}^{2}(4 K(x-\omega t))
$$

which satisfy (1) when $p=1$.

The numerical experiments will be devoted to studying the behaviour of the various quantities appearing in Theorem 5.1, We let

$\eta_{i}=\left(\int_{0}^{t^{n}}\left\|\mathcal{E}_{i}(t)\right\|^{2} d s\right)^{1 / 2}, i=1,2,3 ; \quad \eta_{4}=\left(\sum_{j=0}^{n}\left\|\mathcal{E}_{4}^{j}\right\|^{2}\right)^{1 / 2}, \quad \eta_{t o t}=\left(\sum_{i=1}^{4} \eta_{i}^{2}\right)^{1 / 2}$.

We begin our numerical experiments with a study of the rate of decrease of the (total) a posteriori error indicator $\eta_{t o t}$. In particular, we would like to show that it decreases at the rate of $O(\kappa)$ just as the predicted rate of decrease of the error $\left\|u(T)-u^{N}\right\|$ where $\kappa N=T$. In order to render the spatial errors very small, we chose $M=500$ and $q=5$. Table 1 shows that the rate of decrease of $\eta_{t o t}$ as well as the $L^{2}$ error at $T=1$ decrease at the rate of $O(\kappa)$, with the rate improving as $\kappa$ becomes smaller.

TABLE 1 . Solitary wave solution, $T=1, p=1, \epsilon=.0001, M=$ $500, q=5$

\begin{tabular}{|c|c|c|c|c|c|c|c|c|}
\hline$N$ & $\eta_{1}$ & $\eta_{2}$ & $\eta_{3}$ & $\eta_{4}$ & $\eta_{\text {tot }}$ & rate & $\left\|u(T)-u^{N}\right\|$ & rate \\
\hline 100 & $2.74 \mathrm{E}-02$ & $3.55 \mathrm{E}-07$ & $2.05 \mathrm{E}-02$ & $8.50 \mathrm{E}-02$ & $9.16 \mathrm{E}-02$ & & $1.85 \mathrm{E}-01$ & \\
\hline 200 & $9.28 \mathrm{E}-03$ & $4.75 \mathrm{E}-07$ & $7.29 \mathrm{E}-03$ & $5.29 \mathrm{E}-02$ & $5.42 \mathrm{E}-02$ & 0.757 & $1.33 \mathrm{E}-01$ & .476 \\
\hline 400 & $2.86 \mathrm{E}-03$ & $5.80 \mathrm{E}-07$ & $2.30 \mathrm{E}-03$ & $3.07 \mathrm{E}-02$ & $3.10 \mathrm{E}-02$ & 0.806 & $8.29 \mathrm{E}-02$ & .682 \\
\hline 800 & $8.16 \mathrm{E}-04$ & $6.55 \mathrm{E}-07$ & $6.63 \mathrm{E}-04$ & $1.69 \mathrm{E}-02$ & $1.69 \mathrm{E}-02$ & 0.875 & $4.67 \mathrm{E}-02$ & .828 \\
\hline 1600 & $2.20 \mathrm{E}-04$ & $7.01 \mathrm{E}-07$ & $1.79 \mathrm{E}-04$ & $8.88 \mathrm{E}-03$ & $8.88 \mathrm{E}-03$ & 0.928 & $2.48 \mathrm{E}-02$ & .913 \\
\hline 3200 & $5.72 \mathrm{E}-05$ & $7.27 \mathrm{E}-07$ & $4.88 \mathrm{E}-05$ & $4.57 \mathrm{E}-03$ & $4.57 \mathrm{E}-03$ & 0.958 & $1.28 \mathrm{E}-02$ & .954 \\
\hline
\end{tabular}


The results encapsulated in the above table also show that as $\kappa$ decreases $\eta_{4}$ converges to $\eta_{t o t}$. This has practical value in that among all the indicators $\eta_{4}$ is the least expensive to evaluate.

In the next two sets of four plots, we show the dependence on $t$ of the six quantities $\eta_{i}, i=1, \ldots, 4, \eta_{t o t}$ and $\left\|u\left(t^{n}\right)-u^{n}\right\|$. We have not recorded the terms $\left\|u^{n}-\hat{U}\left(t^{n}\right)\right\|$ that also appear in that estimate since in all the experiments performed they were negligible in comparison with the above six terms.
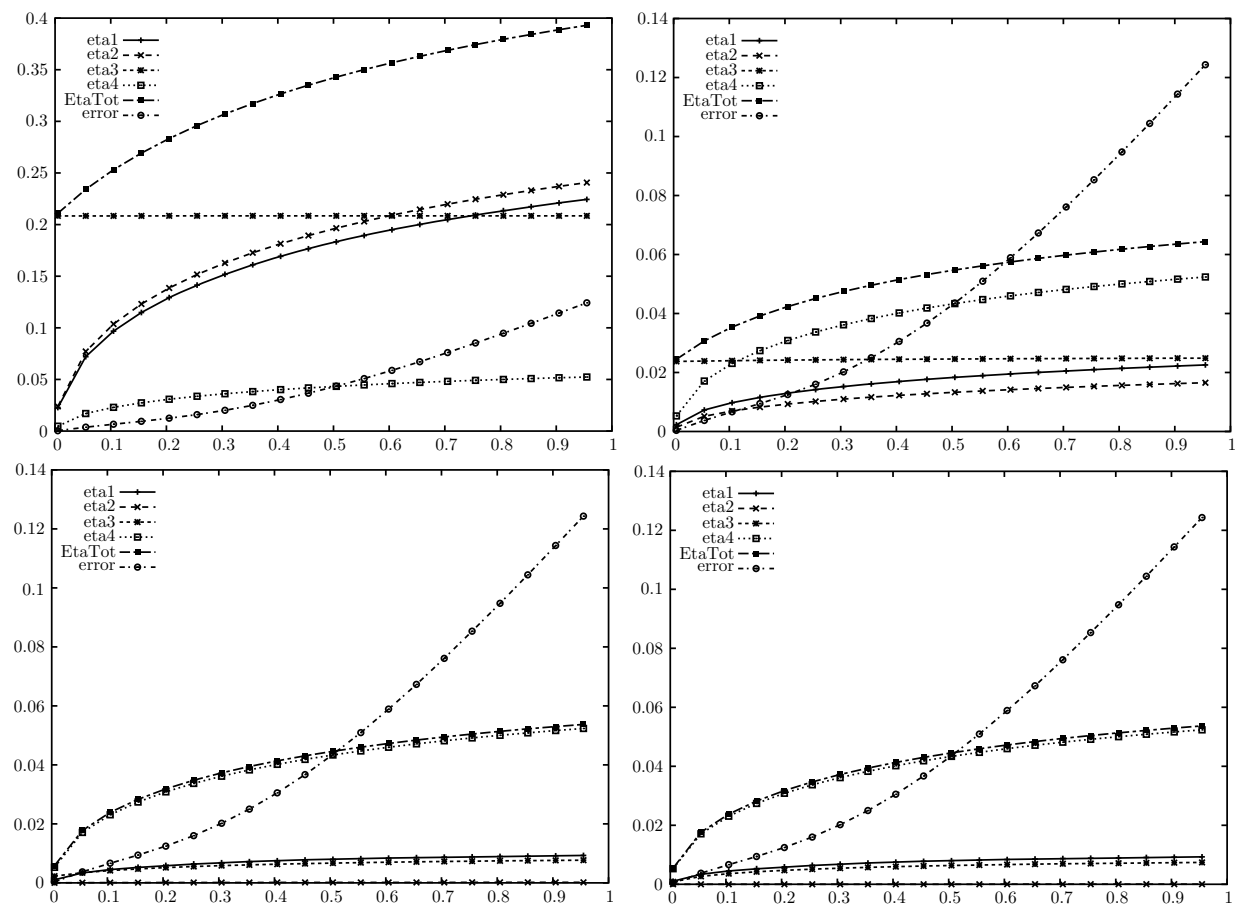

Figure 1. Solitary wave: $A=1, p=1, \epsilon=10^{-4}, M=200$, $N=200, T=1$. Top left: $q=2$, top right: $q=3$, bottom left: $q=4$, bottom right: $q=5$.

The motivation behind the choices of numerical parameters is this: The sequence $q=2,3,4,5$ is considered to correspond to an increasing degree of spatial accuracy whereas the value of $\kappa=.005$ in Figure 1 corresponds to low temporal accuracy with $\kappa=.002$ in Figure 2 corresponding to higher temporal accuracy.

We make the following observations.

(1) For $q=2$ and $q=3$, the term $\mathcal{D}\left(u_{M}^{n+1}-u^{n+1}\right)$ in $\eta_{3}$ is relatively large over the first one or two steps and then becomes very small by several orders of magnitude. This explains the fact that $\eta_{3}$ appears to be constant in time. We do not yet have an explanation for this admittedly peculiar beheviour, except that it seems to occur only for $q=2$ and $q=3$, appears to be independent of $M$ and the test function used (see Figure 3). One reasonable approach would be to take $\eta_{3}$ into account only after the first two steps or even to ignore it altogether. 
(2) From the point of view of effectivity indices, $\eta_{t o t}$ and the actual errors are within a factor of 2 or 3 of each other over the range of integrations considered.

(3) In all cases the $L^{2}$ errors grew at a faster than linear rate. This is not surprising in view of the nonlinear nature of the problem. On the other hand, one observes that all the error indicators appear to grow at a rate proportional to $\sqrt{t^{n}}$. This follows from their definition and the fact that we do not include the exponential term that appears in the estimate (58).

(4) It appears that $\eta_{4}$ is a more reliable error indicator than $\eta_{t o t}$ in the cases $q=2$ and $q=3$. It is therefore tempting to develop a heuristic of using only $\eta_{4}$. This would lead to some computational savings given that $\eta_{4}$ is the least expensive error indicator.
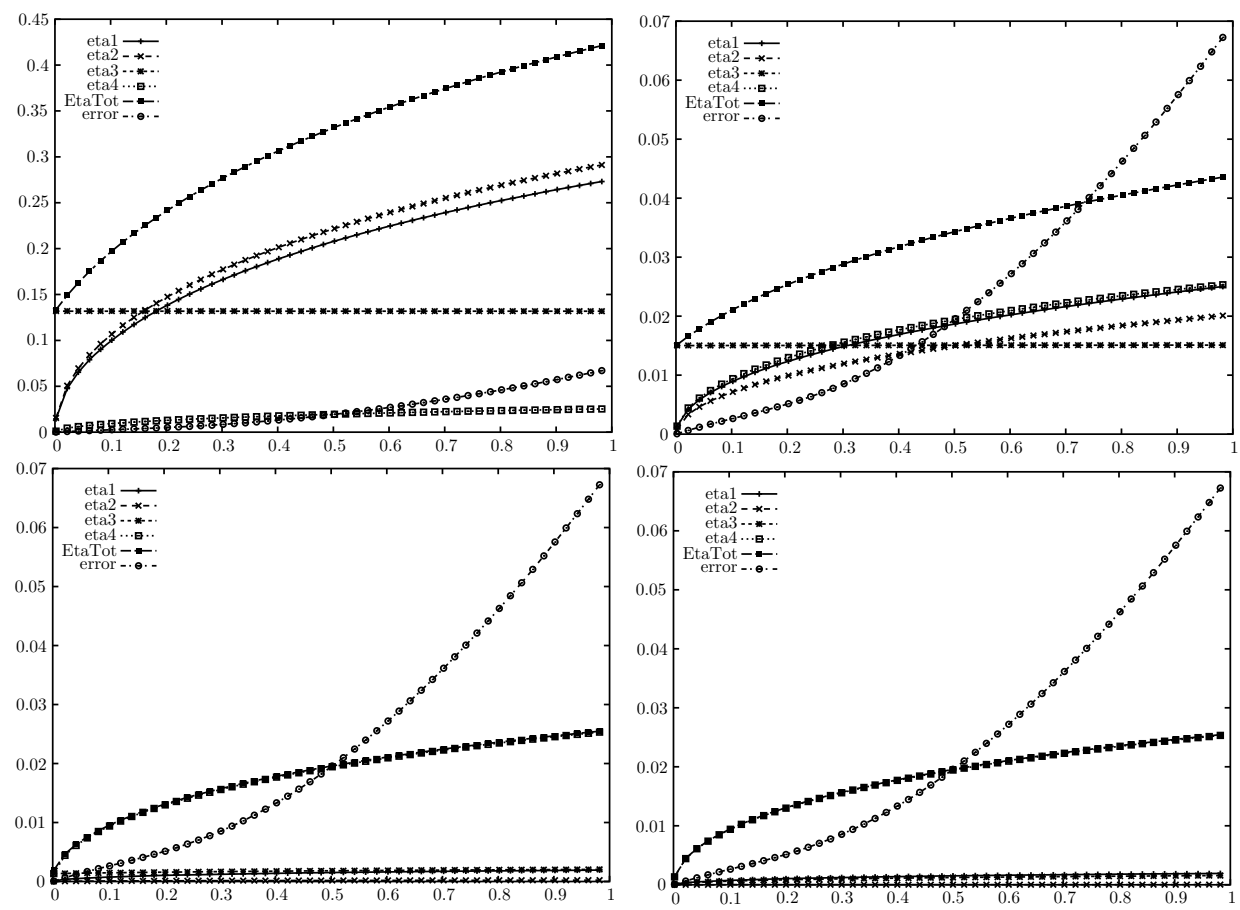

Figure 2. Solitary wave: $A=1, p=1, \epsilon=10^{-4}, M=200$, $N=500, T=1$. Top left: $q=2$, top right: $q=3$, bottom left: $q=4$, bottom right: $q=5$.

Summary and future work. We have constructed a posteriori error indicators and obtained upper bounds for the error as measured in the $L^{2}$ norm. These are the first of their kind for the class of nonlinear dispersive PDE's considered. Yet, there is much that can and should be done. Beyond the extensions to higher order temporal discretizations and the treatment of other nonlinear dispersive equations possessing higher order spatial derivatives, we would like to develop a deeper understanding of the error indicators and their behaviour. In particular, obtaining separate bounds for the spatial and temporal components of the error would be especially interesting. 

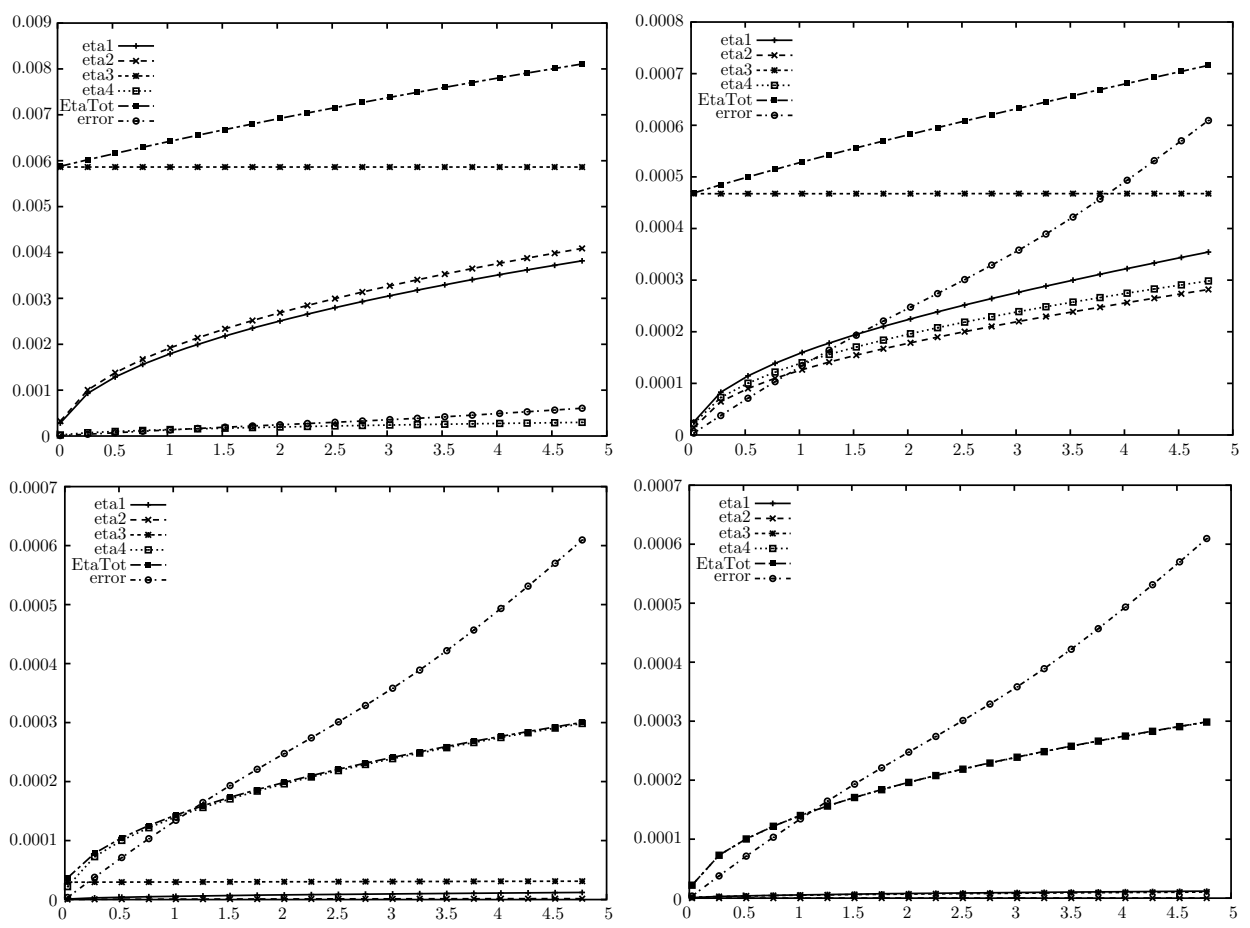

Figure 3. Cnoidal wave: $a=.1148, K=2.5781, \omega=.03403$, $\epsilon=10^{-4}, M=100, N=200, T=5$. Top left: $q=2$, top right: $q=3$, bottom left: $q=4$, bottom right: $q=5$.

By enabling independent control of the spatial mesh and temporal stepsize, effective adaptive strategies and algorithms can be implemented. Last but not least, we would like to obtain computable lower bounds for the errors.

\section{REFERENCES}

[1] K. Abe and O. Inoue, Fourier expansion solution of the Korteweg-de Vries equation, J. Comput. Phys. 34 (1980), no. 2, 202-210, DOI 10.1016/0021-9991(80)90105-9. MR559996 (81a:65113)

[2] R. A. Adams, Sobolev Spaces, Academic Press [A subsidiary of Harcourt Brace Jovanovich, Publishers], New York-London, 1975. Pure and Applied Mathematics, Vol. 65. MR0450957 (56 \#9247)

[3] G. Akrivis, C. Makridakis, and R. H. Nochetto, A posteriori error estimates for the CrankNicolson method for parabolic equations, Math. Comp. 75 (2006), no. 254, 511-531, DOI 10.1090/S0025-5718-05-01800-4. MR2196979(2007a:65114)

[4] M. E. Alexander and J. L. Morris, Galerkin methods applied to some model equations for non-linear dispersive waves, J. Comput. Phys. 30 (1979), no. 3, 428-451, DOI 10.1016/00219991(79)90124-4. MR530003 (80c:76006)

[5] G. A. Baker, V. A. Dougalis, and O. A. Karakashian, Convergence of Galerkin approximations for the Korteweg-de Vries equation, Math. Comp. 40 (1983), no. 162, 419-433, DOI 10.2307/2007524. MR689464 (84f:65072)

[6] G. A. Baker, W. N. Jureidini, and O. A. Karakashian, Piecewise solenoidal vector fields and the Stokes problem, SIAM J. Numer. Anal. 27 (1990), no. 6, 1466-1485, DOI 10.1137/0727085. MR.1080332(91m:65246) 
[7] E. Bänsch, F. Karakatsani, and Ch. Makridakis, A posteriori error control for fully discrete Crank-Nicolson schemes, SIAM J. Numer. Anal. 50 (2012), no. 6, 2845-2872, DOI 10.1137/110839424. MR 3022245

[8] T. B. Benjamin, J. L. Bona, and J. J. Mahony, Model equations for long waves in nonlinear dispersive systems, Philos. Trans. Roy. Soc. London Ser. A 272 (1972), no. 1220, 47-78. MR0427868 (55 \#898)

[9] J. Bona, Model equations for waves in nonlinear dispersive systems, Proceedings of the International Congress of Mathematicians (Helsinki, 1978), Acad. Sci. Fennica, Helsinki, 1980, pp. 887-894. MR562704 (83b:35103)

[10] J. Bona. On solitary waves and their role in the evolution of long waves, in H. Amann, N. Bazley, and K. Kirchgässner, editors, Applications of Nonlinear Analysis in the Physical Sciences, pages 183-205. Pitman: London, 1981.

[11] J. L. Bona, H. Chen, O. Karakashian, and Y. Xing, Conservative, discontinuous Galerkinmethods for the generalized Korteweg-de Vries equation, Math. Comp. 82 (2013), no. 283, 1401-1432, DOI 10.1090/S0025-5718-2013-02661-0. MR3042569

[12] S. C. Brenner and L. R. Scott, The Mathematical Theory of Finite Element Methods, 2nd ed., Texts in Applied Mathematics, vol. 15, Springer-Verlag, New York, 2002. MR.1894376 (2003a:65103)

[13] Y. Cheng and C.-W. Shu, A discontinuous Galerkin finite element method for time dependent partial differential equations with higher order derivatives, Math. Comp. 77 (2008), no. 262, 699-730, DOI 10.1090/S0025-5718-07-02045-5. MR.2373176 (2008m:65252)

[14] B. Cockburn and C.-W. Shu, TVB Runge-Kutta local projection discontinuous Galerkin finite element method for conservation laws. II. General framework, Math. Comp. 52 (1989), no. 186, 411-435, DOI 10.2307/2008474. MR983311 (90k:65160)

[15] V. A. Dougalis and O. A. Karakashian, On some high-order accurate fully discrete Galerkin methods for the Korteweg-de Vries equation, Math. Comp. 45 (1985), no. 172, 329-345, DOI 10.2307/2008128. MR804927 (86m:65118)

[16] B. Fornberg and G. B. Whitham, A numerical and theoretical study of certain nonlinear wave phenomena, Philos. Trans. Roy. Soc. London Ser. A 289 (1978), no. 1361, 373-404, DOI 10.1098/rsta.1978.0064. MR497916(80i:35156)

[17] E. H. Georgoulis, O. Lakkis, and J. M. Virtanen, A posteriori error control for discontinuous Galerkin methods for parabolic problems, SIAM J. Numer. Anal. 49 (2011), no. 2, 427-458, DOI 10.1137/080722461. MR2784879(2012d:65205)

[18] O. Karakashian and W. McKinney, On optimal high-order in time approximations for the Korteweg-de Vries equation, Math. Comp. 55 (1990), no. 192, 473-496, DOI 10.2307/2008429. MR 1035935 (92h:65172)

[19] I. Kyza and C. Makridakis, Analysis for time discrete approximations of blow-up solutions of semilinear parabolic equations, SIAM J. Numer. Anal. 49 (2011), no. 1, 405-426, DOI 10.1137/100796819. MR2783232 (2012d:65185)

[20] O. Lakkis and C. Makridakis, Elliptic reconstruction and a posteriori error estimates for fully discrete linear parabolic problems, Math. Comp. 75 (2006), no. 256, 1627-1658, DOI 10.1090/S0025-5718-06-01858-8. MR2240628(2007e:65122)

[21] O. Lakkis and T. Pryer, Gradient recovery in adaptive finite-element methods for parabolic problems, IMA J. Numer. Anal. 32 (2012), no. 1, 246-278, DOI 10.1093/imanum/drq019. MR2875251

[22] C. Makridakis and R. H. Nochetto, Elliptic reconstruction and a posteriori error estimates for parabolic problems, SIAM J. Numer. Anal. 41 (2003), no. 4, 1585-1594, DOI 10.1137/S0036142902406314. MR2034895(2004k:65157)

[23] C. Makridakis and R. H. Nochetto, A posteriori error analysis for higher order dissipative methods for evolution problems, Numer. Math. 104 (2006), no. 4, 489-514, DOI 10.1007/s00211-006-0013-6. MR2249675 (2008b:65114)

[24] J.-C. Saut, Applications de l'interpolation non linéaire à des problèmes d'évolution non linéaires (French), J. Math. Pures Appl. (9) 54 (1975), 27-52. MR0454374 (56 \#12625)

[25] H. Schamel and K. Elsässer, The application of the spectral method to nonlinear wave propagation, J. Computational Phys. 22 (1976), no. 4, 501-516. MR0449164 (56 \#7469)

[26] T. R. Taha and M. J. Ablowitz, Analytical and numerical aspects of certain nonlinear evolution equations. III. Numerical, Korteweg-de Vries equation, J. Comput. Phys. 55 (1984), no. 2, 231-253, DOI 10.1016/0021-9991(84)90004-4. MR762364(86e:65128c) 
[27] T. Taha and M. Ablowitz, Analytical and numerical aspects of certain nonlinear evolution equations. IV, Numerical, modified Korteweg-de Vries equation. J. Comp. Phys., 77:540-548, 1988.

[28] F. Tappert, Numerical solution of the Korteweg-de Vries equation and its generalizations by the split-step Fourier method. In A. C. Newell, editor, Nonlinear Wave Motion, Lectures in Applied Mathematics, pages 215-216. Amer. Math. Soc., Providence, R.I., 1974.

[29] A. C. Vliegenthart, On finite-difference methods for the Korteweg-de Vries equation, J. Engrg. Math. 5 (1971), 137-155. MR0363153(50 \#15591)

[30] L. B. Wahlbin, A dissipative Galerkin method for the numerical solution of first order hyperbolic equations, Mathematical aspects of finite elements in partial differential equations (Proc. Sympos., Math. Res. Center, Univ. Wisconsin, Madison, Wis., 1974), Math. Res. Center, Univ. of Wisconsin-Madison, Academic Press, New York, 1974, pp. 147-169. Publication No. 33. MR0658322(58 \#31929)

[31] R. Winther, A conservative finite element method for the Korteweg-de Vries equation, Math. Comp. 34 (1980), no. 149, 23-43, DOI 10.2307/2006219. MR.551289 (81a:65108)

[32] Y. Xu and C.-W. Shu, Error estimates of the semi-discrete local discontinuous Galerkin method for nonlinear convection-diffusion and KdV equations, Comput. Methods Appl. Mech. Engrg. 196 (2007), no. 37-40, 3805-3822, DOI 10.1016/j.cma.2006.10.043. MR.2340006 (2009e:65139)

[33] J. Yan and C.-W. Shu, A local discontinuous Galerkin method for KdV type equations, SIAM J. Numer. Anal. 40 (2002), no. 2, 769-791 (electronic), DOI 10.1137/S0036142901390378. MR.1921677 (2003e:65181)

Department of Mathematics, The University of Tennessee, Knoxville, Tennessee 37996

E-mail address: ohannes@math.utk.edu

Department of Applied Mathematics, The University of Crete, Heraklion, Greece AND - IACM-FORTH, 70013 Heraklion-Crete, GreECE

E-mail address: makr@tem.uoc.gr

Current address: School of Mathematical and Physical Sciences, University of Sussex, Brighton, BN1 9QH, United Kingdom 